\title{
The stromal composition of mast cell aggregates in systemic mastocytosis
}

\author{
April Chiu ${ }^{1}$, Nahid M Nanaji ${ }^{2}$, Magdalena Czader ${ }^{3}$, Gabriela Gheorghe $^{3}$, \\ Daniel M Knowles ${ }^{1}$, Amy Chadburn ${ }^{1, *}$ and Attilio Orazi ${ }^{1}$ \\ ${ }^{1}$ Department of Pathology and Laboratory Medicine, Weill Cornell Medical College, New York, NY, USA; \\ ${ }^{2}$ Department of Pathology, University of Maryland School of Medicine, Baltimore, MD, USA and ${ }^{3}$ Department \\ of Pathology and Laboratory Medicine, Indiana University School of Medicine, Indianapolis, IN, USA
}

\begin{abstract}
Systemic mastocytosis is a stem cell disorder characterized histologically by the presence of multifocal compact aggregates of mast cells in at least one extracutaneous organ with or without evidence of skin lesions. The mast cell aggregates are accompanied by fibrosis, which is often significant. However, in spite of its frequent occurrence and severity, little is known about its characteristics. In this study, we evaluated the composition of the fibrotic mast cell aggregates by studying eight bone marrow biopsies and two spleens involved by systemic mastocytosis, and compared the findings with those observed in other fibrotic bone marrow disorders such as primary myelofibrosis and metastatic malignancy. Histochemistry and immunohistochemistry were used to evaluate: (a) extracellular matrix (reticulin, trichrome, collagen IV, laminin); (b) stromal reticulum cells (low-affinity nerve growth factor receptor); (c) presence of myofibroblastic differentiation (smooth muscle actin) and (d) microvessel density (CD34). We found that all cases showed marked reticulin and collagen fibrosis. However, unlike primary myelofibrosis and metastatic malignancy, which are usually associated with increased low-affinity nerve growth factor receptor positivity, its expression was low in all cases of systemic mastocytosis. Myofibroblastic differentiation was only focally detected in two of eight bone marrow biopsies. In all cases, the systemic mastocytosis lesions were largely devoid of type IV collagen and laminin. The latter findings were in contrast with those seen in cases of primary myelofibrosis and metastatic malignancy where smooth muscle actin, collagen IV and laminin were expressed in most cases. Also in contrast with the other two conditions, only minimal vascularity was detectable within the fibrotic mast cell lesions. These findings indicate that systemic mastocytosis exhibits a distinct pattern of stromal change, and suggest that the fibrogenetic mechanism in systemic mastocytosis is most likely different from that of other bone marrow neoplasms which are also associated with fibrosis.
\end{abstract}

Modern Pathology (2009) 22, 857-865; doi:10.1038/modpathol.2009.53; published online 24 April 2009

Keywords: mastocytosis; mast cells; fibrosis; bone marrow; stroma; microvessels

Systemic mastocytosis is a stem cell disorder characterized by a pathological accumulation of clonal mast cells in one or more organ systems. The diagnosis of systemic mastocytosis is usually established by bone marrow examination, although diagnostic material also can be obtained from other sites. Bone marrow histology typically shows multiple lesions, which consist of compact clusters or cohesive mast cell aggregates, often occurring next to the trabecular bone and/or surrounding vascular

Correspondence: Dr A Orazi, MD, Department of Pathology and Laboratory Medicine, Weill Cornell Medical College, 525 E. 68th Street, Room ST-702B, Starr 715, New York, NY 10065, USA.

E-mail: ato9002@med.cornell.edu

${ }^{*}$ Current address: Department of Pathology, Northwestern University, Chicago, IL, USA

Received 15 January 2009; revised and accepted 2 March 2009; published online 24 April 2009 structures. ${ }^{1,2}$ These multifocal mast cell aggregates in the bone marrow and/or other extracutaneous organs represent the major criterion for the diagnosis of systemic mastocytosis according to the 2008 World Health Organization classification. ${ }^{2}$ The diagnosis of systemic mastocytosis requires, besides fulfilling the major criterion, at least one of the minor criteria (or in the absence of the major criterion, at least three of the minor ones). The minor criteria include: (1) atypical morphology, including spindle shape, by $>25 \%$ of the mast cells; (2) identification of an activating mutation at codon 816 of KIT; (3) aberrant expression of CD2 and/or CD25 by the mast cells; and (4) a persistently elevated level of serum total tryptase $(>20 \mathrm{ng} / \mathrm{ml}){ }^{2}$ Histologically, the mast cell lesions are characteristically associated with fibrosis, which is often significant; the adjacent trabecular bone may be variably osteosclerotic. The mast cells produce a 
variety of fibrogenic cytokines, including plateletderived growth factor, transforming growth factor- $\beta$ (TGF- $\beta$ ), and basic fibroblast growth factor. ${ }^{3-5}$ These cytokines are involved in numerous inflammatory and neoplastic conditions in which marrow fibrosis is encountered and are thus not specific to mast cells. In addition, mast cell tryptase, which has been shown to be a powerful fibroblast mitogen, ${ }^{6}$ is thought to play an important fibrogenic role in systemic mastocytosis. Both mast cell tryptase and histamine, another substance produced by mast cells, are capable of stimulating synthesis of type I collagen by fibroblasts. ${ }^{6-9}$ However, in spite of these data, there is very little information available regarding either the stromal cell or the extracellular matrix composition of the fibrotic areas involved by systemic mastocytosis.

In this study, we evaluated the histochemical and immunohistochemical characteristics of extracellular matrix and stromal cell components by studying bone marrow and spleen specimens of patients with systemic mastocytosis and compared the findings with those observed in fibrotic bone marrows of patients with primary myelofibrosis and metastatic malignancy, conditions which are also often associated with significant marrow fibrosis.

\section{Materials and methods}

\section{Tissue Samples}

Paraffin-embedded tissue blocks of eight bone marrow biopsies and two spleens of 10 patients diagnosed with systemic mastocytosis were obtained from the files of the Department of Pathology and Laboratory Medicine, Weill Cornell Medical College/New York Presbyterian Hospital (New York, NY), and Indiana University School of Medicine/ Clarian Health Partners (Indianapolis, IN). In addition, bone marrow biopsies of patients diagnosed with fibrotic primary myelofibrosis (5) and bone marrow biopsies involved by metastatic malignancy associated with fibrosis (4), were evaluated for comparison. Both spleen specimens were formalinfixed, whereas the bone marrow biopsies were fixed in either formalin or Bouin's fixative. Four-micron tissue sections were prepared from the paraffin blocks for routine morphologic evaluation and for immunohistochemistry. IRB approval was obtained at both institutions.

\section{Histochemistry}

Histochemical staining for reticulin (reactivity with all types of fibers) and trichrome (reactivity with collagen fibers) was performed using an automated stainer (Leica Microsystems, Bannockburn, IL, USA) according to the manufacturer's protocol with mild modifications.

\section{Immunohistochemistry}

Immunohistochemical studies were performed using a Bond-Max autostainer (Vision BioSystems, Hingham, MA, USA) using a polymer-define peroxidase detection system after antigen retrieval with epitope retrieval solution 2 (Vision BioSystems). Monoclonal antibodies against the following antigens were used to evaluate the bone marrow stroma: collagen type IV (CIV 22), smooth muscle actin (1A4), low-affinity nerve growth factor receptor (ME20.4) (Dako North America Inc., Carpinteria, CA), laminin (LAM89; Vision BioSystems Novocastra, Newcastle-uponTyne, United Kingdom), and CD34 (Qbend10; Biogenex Laboratories, San Ramon, CA).

\section{Evaluation of Extracellular Matrix and Stromal Cell Composition}

The extracellular matrix was evaluated by histochemical staining for reticulin (reactivity with all types of fibers) and trichrome (reactivity with collagen fibers), as well as immunohistochemistry for the expression of type IV collagen and laminin. The degree of reticulin fibrosis was graded according to the European consensus scoring system $(0$ to $3+)$ originally proposed for grading of fibrosis in patients with primary myelofibrosis. ${ }^{10}$ Collagen fibrosis was graded either positive or negative. Immunohistochemical staining for low-affinity nerve growth factor receptor was performed to identify the adventitial reticular cells, and smooth muscle actin to identify myofibroblastic differentiation within the bone marrow stroma. The positivity cells for each marker was determined semi-quantitatively as focally positive $(\mathrm{f}+)$, positive $(+)$, and strongly positive $(++)$.

Microvessel density was assessed by immunohistochemical staining for CD34. The number of CD34 positive microvessels within the mast cell lesions was compared with that of the adjacent uninvolved marrow tissue and designated as increased or not increased. The evaluation was performed by using a $\times 40$ lens by averaging the findings of all fibrotic areas present in one section compared with the non fibrotic bone marrow fields (a minimum of five $\times 40$ fields per section were analyzed). Similar criteria for assessing extracellular matrix, cellular composition and microvessel density were used for the assessment of the primary myelofibrosis and metastatic malignancy marrows.

\section{Results}

\section{Clinical and Pathological Features}

The clinical and pathological characteristics of the cases are summarized in Table 1. The patients were diagnosed with systemic mastocytosis according to the World Health Organization criteria. There were three women and seven men ranging in age from 37 
Table 1 Clinical characteristics of systemic mastocytosis cases

\begin{tabular}{llllll}
\hline Case no. & Age $^{\mathrm{a}}$ & Sex & Site $^{\mathrm{b}}$ & AHNMD $^{\mathrm{c}}$ & Cytogenetics $^{\mathrm{d}}$ \\
\hline 1 & 47 & M & BM & None & Normal \\
3 & 50 & F & BM & None & ND \\
4 & 47 & M & BM & None & Normal \\
5 & 67 & M & BM & AML & -7 \\
6 & 75 & F & BM & PV & Normal \\
7 & 37 & F & BM & None & ND \\
8 & 50 & M & BM & RAEB-1 & Failed \\
9 & 72 & M & Spleen & MDS/MPD & Normal \\
10 & 81 & M & Spleen & None & ND \\
\hline
\end{tabular}

${ }^{\mathrm{a}}$ Mean age 60.2 (range 37-81) years.

${ }^{\mathrm{b}} \mathrm{BM}$, bone marrow.

${ }^{\mathrm{c}}$ AHNMD, associated clonal hematological non-mast-cell lineage disease; AML, acute myeloid leukemia; PV, polycythemia vera; RAEB-1, refractory anemia with excess blasts-1; MDS/MPD, myelodysplastic syndrome/myeloproliferative disease.

${ }^{\mathrm{d}} \mathrm{ND}$, not done; -7 , monosomy 7 .

Table 2 Histochemical and immunohistochemical results

Case no. Reticulin Trichrome Coll IV Laminin SMA LNGFR CD34

\begin{tabular}{|c|c|c|c|c|c|c|c|}
\hline \multicolumn{8}{|c|}{$S M$} \\
\hline 1 & $3+$ & + & - & - & $\mathrm{f}_{+}$ & - & - \\
\hline 2 & ND & ND & - & - & - & - & ND \\
\hline 3 & $3+$ & + & - & - & - & - & - \\
\hline 4 & $3+$ & + & - & - & - & - & - \\
\hline 5 & $3+$ & + & - & - & - & - & - \\
\hline 6 & $3+$ & + & - & - & - & - & - \\
\hline 7 & $3+$ & + & - & - & - & - & ND \\
\hline 8 & $3+$ & + & - & - & + & + & ND \\
\hline 9 & $3+$ & + & - & - & - & - & ND \\
\hline 10 & $3+$ & + & - & - & - & - & - \\
\hline \multicolumn{8}{|c|}{$P M F$} \\
\hline 1 & $3+$ & + & + & + & + & ++ & $\uparrow$ \\
\hline 2 & $3+$ & + & - & - & + & - & $\uparrow$ \\
\hline 3 & $3+$ & + & - & - & $\mathrm{f}+$ & + & $\uparrow$ \\
\hline 4 & $3+$ & + & + & + & + & ++ & $\uparrow$ \\
\hline 5 & $3+$ & + & - & - & + & ++ & $\uparrow$ \\
\hline \multicolumn{8}{|c|}{$M M A L$} \\
\hline 1 & $3+$ & + & + & + & ++ & + & $\uparrow$ \\
\hline 2 & $3+$ & + & - & $\mathrm{f}+$ & + & + & $\uparrow$ \\
\hline 3 & $\mathrm{f}+$ & + & - & $\mathrm{f}+$ & + & + & $\uparrow$ \\
\hline 4 & $3+$ & + & + & + & - & + & $\uparrow$ \\
\hline
\end{tabular}

SM, systemic mastocytosis; PMF, primary myelofibrosis; MMAL, metastatic malignancy; ND, not done; Coll IV, collagen IV; SMA, smooth muscle actin; LNGFR, low affinity nerve growth factor receptor; f+, focally positive; $\uparrow$, increased vessels.

to 81 years (mean, 60.2 years). Four patients had an associated clonal hematological non-mast-cell lineage disease, whereas the remaining patients had no associated hematologic malignancy. The hematological malignancies associated with systemic mastocytosis are shown in Table 2.

\section{Morphology}

Examination of the bone marrow sections showed multiple focal dense mast cell aggregates character- istic of systemic mastocytosis. The mast cell aggregates were often paratrabecular or perivascular in location and associated with fibrosis that was often dense, relatively acellular and poorly vascularized (Figure 1a). The trabecular bone often showed mild to pronounced osteosclerosis. The splenic capsule was significantly thickened. Examination of the spleen sections showed mast cell infiltrates associated with fibrosis (Figure 1d) scattered throughout the splenic parenchyma and/or involving the periphery of the white pulp or septa and trabeculae. The cases of primary myelofibrosis and metastatic malignancy showed histologic findings fully representative of the two disease groups, and degrees of fibrosis, which by histochemical stains (not shown) corresponded to an MF-3 grade (Figure 1e and f).

\section{Extracellular Matrix}

Reticulin and trichrome histochemical stains were performed in all cases with the exception of one bone marrow case in which the block was exhausted (Table 2). All the evaluated cases (systemic mastocytosis, primary myelofibrosis, and metastatic malignancy) showed, in the involved areas, MF-3 fibrosis by reticulin staining associated with collagen fibrosis as determined by trichrome staining (Figure $1 \mathrm{~b}$ and $\mathrm{c}$ ). The spleen cases involved by systemic mastocytosis showed comparable results. Immunohistochemical staining for extracellular matrix and stromal cells was performed in all cases. In systemic mastocytosis, there was no significant expression of collagen IV or laminin within the lesions except in association with rare microvessels (Figure 2a and $\mathrm{b}$ ).

In contrast, all four bone marrow biopsies involved by metastatic malignancy showed an increase in either type IV collagen and/or laminin stromal deposition (Figure $2 \mathrm{~d}$ and e), whereas two of the five bone marrow biopsies involved by primary myelofibrosis showed an increased expression of both type IV collagen and laminin (Figure $2 \mathrm{~g}$ and $\mathrm{h}$ ). In the remaining three primary myelofibrosis cases, no significant expression of either marker was detected.

\section{Stromal Cell Composition}

Smooth muscle actin expression was detected in only two of the eight bone marrows and in neither of the two spleens with systemic mastocytosis (Table 2). In one case (case 1), the smooth muscle actin expression was only focal, whereas in the second case (case 9) the expression was more pronounced. Low-affinity nerve growth factor receptor expression was decreased in the fibrotic lesion of systemic mastocytosis in all but one case (case 9), whereas the uninvolved marrow surrounding the fibrotic mast cell lesion showed a normal to mildly increased number of adventitial reticular 

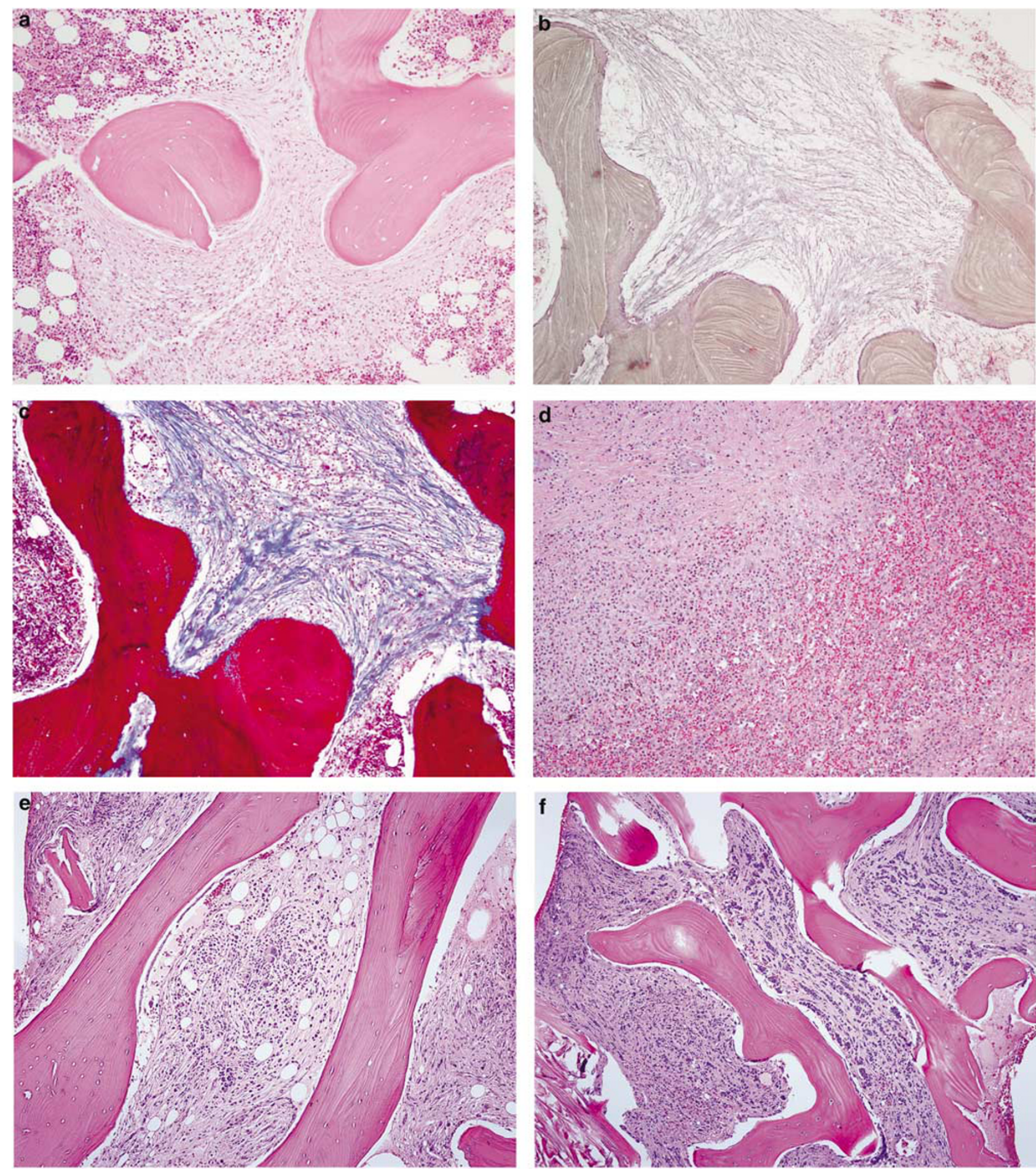

Figure 1 Bone marrow biopsy and spleen showing involvement by systemic mastocytosis. (a) A large paratrabecular/intertrabecular mast cell aggregate showing extensive fibrosis. (b) Reticulin stain of the same bone marrow biopsy demonstrating marked (MF-3) reticulin fibrosis. (c) Corresponding trichrome stain shows a significant degree of collagen fibrosis. (d) Mast cell lesion in splenic red pulp in a patient with systemic mastocytosis, also characterized by fibrosis. Bone marrow biopsy from (e) a case of PMF and (f) a case of metastatic malignancy (prostatic adenocarcinoma) showing similar degrees of fibrosis, which, by histochemical stains (not shown), corresponded to an MF-3 grade.

cells (Figure 3a and b). In contrast, smooth muscle actin expression was seen in $5 / 5$ primary myelofibrosis (one case showed only focal expression) and $3 / 4$ metastatic malignancy cases, whereas increased low-affinity nerve growth factor receptor expression was detected in 4/5 primary myelofibrosis and in 4/4 metastatic malignancy cases, respectively (Figure 3c-f). 

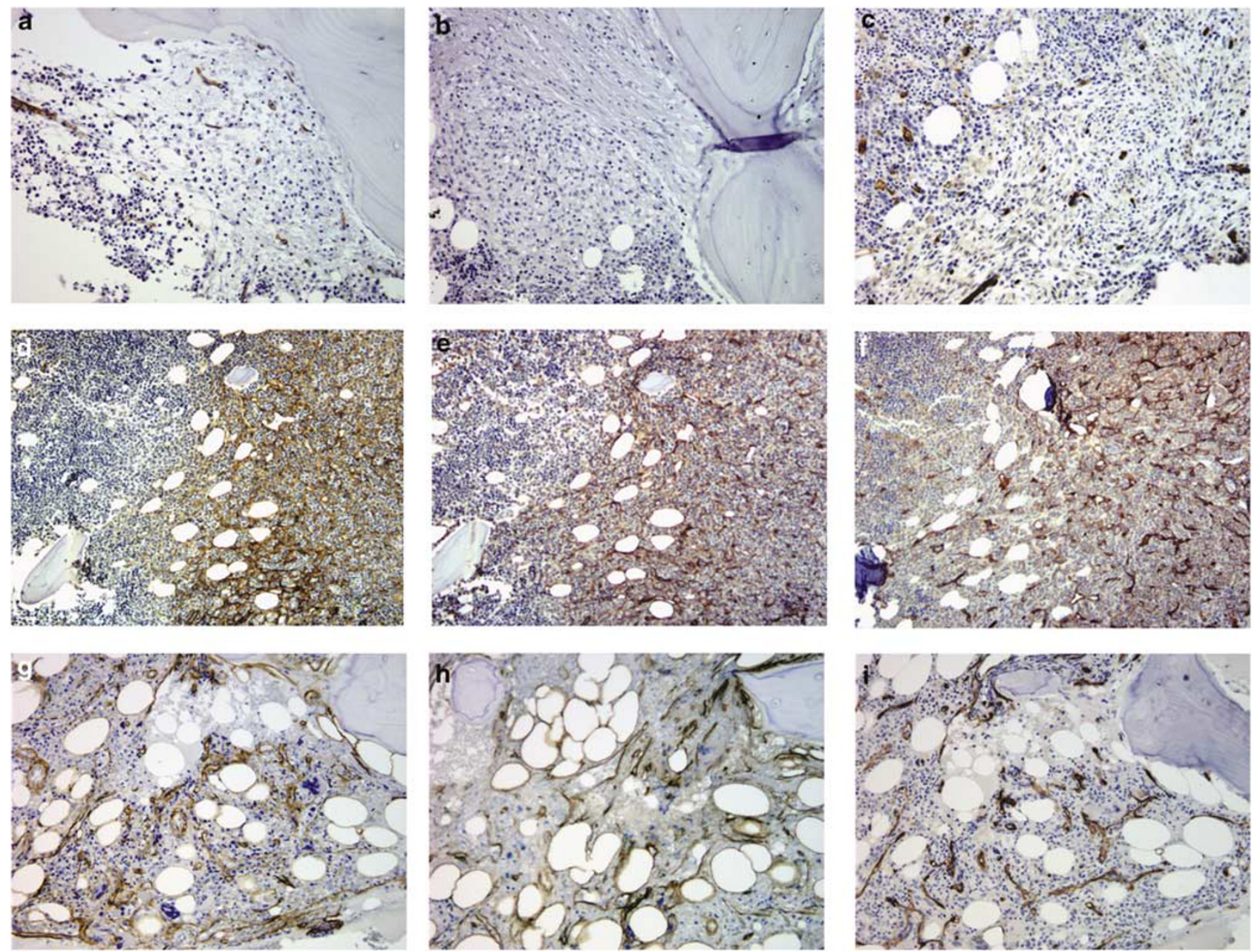

Figure 2 Immunohistochemical staining for type IV collagen, laminin, and CD34. A case of bone marrow biopsy involved by systemic mastocytosis shows no significant increase of either collagen IV (a) or laminin (b), or of microvessel density (c) within the mast cell lesions compared with the surrounding uninvolved bone marrow. In contrast, all bone marrow biopsies involved by metastatic malignancy show an increase in either type IV collagen (d) and/or laminin deposition (e) in the stroma, as well as increased microvessel density (f). A bone marrow biopsy involved by primary myelofibrosis showing increased expression of type IV collagen (g) and laminin (h), as well as increased microvessel density (i).

\section{Microvessel Density by CD34 Immunostaining}

CD34 immunostaining, performed in five bone marrow cases, showed a lower number of microvessels within the systemic mastocytosis lesions than in the surrounding apparently uninvolved bone marrow (Figure 2c). These bone marrow areas contained a variably increased number of microvessels, which were focally more numerous in the three bone marrow cases of systemic mastocytosis associated with clonal hematological non-mast-cell lineage disease. All cases of metastatic malignancy and primary myelofibrosis showed, as expected, a significantly increased microvessel density as highlighted by CD34 staining (Figure $2 \mathrm{f}$ and i).

\section{Discussion}

In this study, we demonstrated that the degree of reticulin and collagen fibrosis within the mast cell lesions of systemic mastocytosis is pronounced and similar to that seen in advanced primary myelofibrosis and in cases of metastatic malignancy associated with fibrosis. However, unlike primary myelofibrosis and metastatic malignancy, which showed increased deposition of basement membrane components collagen IV and laminin, most likely because of increased vascularity, and increased low-affinity nerve growth factor receptor and smooth muscle actin expression, most likely because of an increase in the number of adventitial reticular cells and the presence of myofibroblastic differentiation, respectively, the mast cell lesions lacked these features. In particular, despite the pronounced fibrosis, the mast cell lesions exhibited a marked decrease in low-affinity nerve growth factor receptor expression, most likely due to a decreased number of adventitial reticular cells, with only rare or minimal expression of smooth muscle actin. Thus, our results indicate that the fibrous 
862
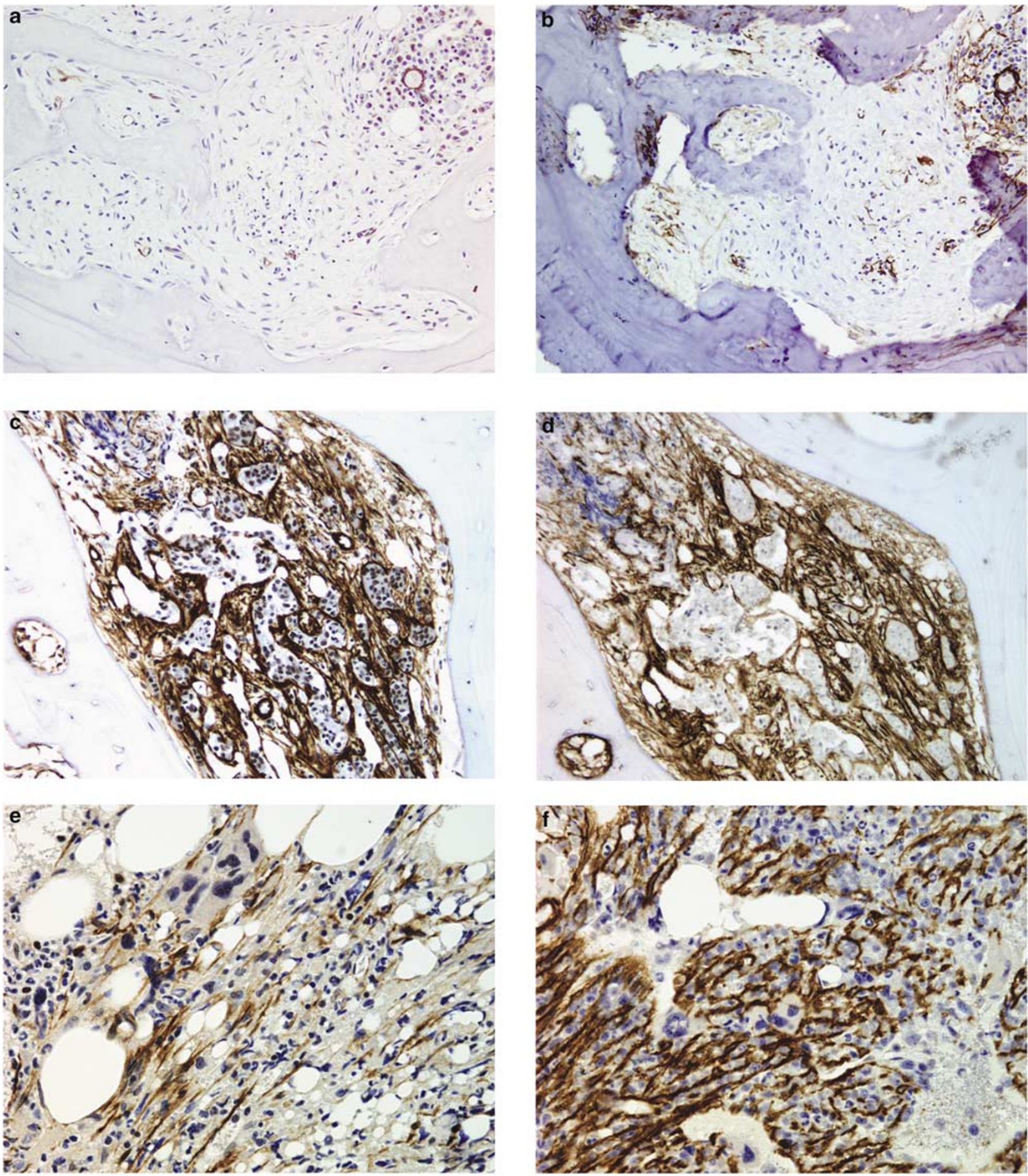

Figure 3 Immunohistochemical staining for smooth muscle actin and low-affinity nerve growth factor receptor. In a bone marrow biopsy involved by systemic mastocytosis, the mast cell aggregate shows neither (a) smooth muscle actin or (b) low-affinity nerve growth factor receptor expression, whereas the uninvolved marrow surrounding the fibrotic mast cell lesion shows normal expression (note the positivity in an arteriole in the surrounding uninvolved marrow for smooth muscle actin). (c) In contrast, a case of primary myelofibrosis shows increased smooth muscle actin expression, signifying the presence of pronounced myofibroblastic differentiation. (d) In the same case of primary myelofibrosis, increased low-affinity nerve growth factor receptor expression is also seen. Similarly, a case of metastatic malignancy in the bone marrow shows increased expression of both smooth muscle actin (e) and low-affinity nerve growth factor receptor (f). 
tissue in systemic mastocytosis is highly collagenized, relatively acellular, and poorly vascularized.

The non-vascular component of the bone marrow microenvironment mostly consists of stromal cells and the extracellular matrix produced by the stromal cells. ${ }^{11-13}$ The stromal cells include adventitial reticular cells. ${ }^{12-14}$ The cytoplasm of the adventitial reticular cells is capable of extending itself in fiberlike strands deep into the hematopoietic cords. These strands provide a meshwork which provides structural support for the vascular sinuses and hematopoietic cells. ${ }^{14}$ Other types of stroma cells include bone marrow macrophages and adipocytes. Myoid cells have also been documented in the bone marrow. ${ }^{15,16}$ They can be easily demonstrated in fetal bone marrow but are rare in adult bone marrow. It is still uncertain whether they represent a distinct subpopulation of reticular cells. ${ }^{15}$ They may develop from adventitial reticular cells which have undergone cytoskeletal remodeling in response to various stimuli. ${ }^{15}$ The extracellular matrix produced by the stromal cells is mostly composed of the structural fibrils such as collagen, reticulin, laminin, and fibronectin, as well as a variety of glycoproteins. ${ }^{17-21}$ Collectively, the various components of the bone marrow microenvironment play an active role in hematopoiesis by producing numerous cytokines, inhibitory factors, adhesion molecules, and contact matrix that promote hematopoietic cell production and osteogenesis. ${ }^{20}$ Alterations in bone marrow stroma can occur in both benign and malignant disorders, ${ }^{21-25}$ including metastatic tumors involving the bone marrow. ${ }^{23}$ They are particularly pronounced and have been better described in myeloid neoplasms associated with bone marrow fibrosis such as primary myelofibrosis. ${ }^{21,22}$

An antibody directed against low-affinity nerve growth factor receptor has been shown to label bone marrow adventitial reticular cells. ${ }^{14,26}$ The adventitial reticular cells are responsible for the deposition of reticulin and collagen III. Upon activation, the adventitial reticular cells may express smooth muscle actin acquiring a myofibroblastic differentiation. In general, there is a correlation between the number of low-affinity nerve growth factor receptor positive adventitial reticular cells and the degree of reticulin fibrosis in primary myelofibrosis and in metastatic malignancy. ${ }^{23}$ Increased expression of low-affinity nerve growth factor receptor, due to the proliferation of adventitial reticular cells usually associated with fibrosis, has also been demonstrated in different subtypes of lymphoma and in reactive marrow. ${ }^{24,25}$ In contrast, the decreased expression of low-affinity nerve growth factor receptor within the fibrotic mast cell lesions of systemic mastocytosis indicates the presence of a smaller number of adventitial reticular cells.

Smooth muscle actin was utilized in this study to identify cells with myofibroblastic differentiation. Smooth muscle actin expression is largely absent in normal bone marrow. ${ }^{15,16}$ In the presence of appropriate stimuli, bone marrow adventitial reticular cells may become activated, differentiating into myofibroblasts. These myofibroblasts become a major source of mature collagen as shown in various neoplasms, including squamous carcinomas ${ }^{27}$ and other malignancies. ${ }^{23,28}$ Stromal smooth muscle actin expression was virtually absent in systemic mastocytosis. Thus, a 'myofibroblastic' stromal phenotype, although common in other bone marrow fibrotic neoplasms, such as metastatic malignancy, is not a common stromal response in bone marrows affected by systemic mastocytosis. It is important to stress, however, that we analyzed tissues at a given point in time whereas the fibrotic process is a dynamic one. Therefore, it is possible that transient myofibroblastic differentiation of bone marrow reticular cells may occur during the early phases of bone marrow involvement with systemic mastocytosis and might not be evident in later stages of the disease, when a bone marrow biopsy is more often performed.

Type IV Collagen, along with laminin, is a major component of basement membrane. ${ }^{18,21,22,29}$ In normal bone marrow, type IV collagen is largely subendothelial in distribution, normally highlighting only vascular walls, whereas adipocytes show only weak pericellular expression. ${ }^{18}$ Collagen IV has been shown to be increased in fibrotic primary myelofibrosis, where it forms continuous sheets of subendothelial basal membrane in advanced primary myelofibrosis. ${ }^{22}$ In contrast, in all of our cases, the fibrotic mast cell lesions were largely devoid of type IV collagen. Laminin was similarly diminished in these lesions. These findings show the lack of basal membrane extracellular matrix proteins, which are mostly associated with vessels, a result which is consistent with the decreased vascularity seen in the fibrotic mast cell lesions. The presence of diminished vascularity was further confirmed by the paucity of CD34-positive microvessels observed in these lesions.

In this study, we have found that despite the marked increase in reticulin fibrosis and the presence of collagen fibrosis, expression of lowaffinity nerve growth factor receptor and extracellular matrix-associated proteins are absent in nearly all cases of systemic mastocytosis. There is also a paucity of smooth muscle actin expression in systemic mastocytosis. The pattern of expression for these stromal markers is thus different from those observed in most cases of primary myelofibrosis and metastatic malignancy. As low-affinity nerve growth factor receptor and type IV collagen are components of the vascular basement membrane, their absence in systemic mastocytosis suggests an overall low vascularity within the fibrotic lesions of systemic mastocytosis. In this regard, immunostaining for CD34, an approach which is commonly used to evaluate microvessel density in neoplastic diseases, ${ }^{30,31}$ confirmed that the fibrotic mast cell lesions are largely devoid of microvessels. 
In contrast, primary myelofibrosis as well as most other myeloid neoplasms, typically show increased microvessel density, ${ }^{32,33}$ a potential target for antiangiogenic treatments (eg, thalidomide or lenalidomide). ${ }^{34,35}$ In contrast, the lack of intralesional microvessels raises serious doubts in regard to the effectiveness of a similar approach for cases of systemic mastocytosis. In this regard, it was recently observed that antiangiogenic therapy in systemic mastocytosis did not result in the normalization of marrow findings including the amount of bone marrow fibrosis in treated patients, ${ }^{36,37}$ or in significant clinical improvement. ${ }^{37}$ Although an increase in bone marrow angiogenesis is reported in the majority of patients with systemic mastocytosis, ${ }^{38,39}$ its pathogenesis is unclear and may not be directly linked to the mast cells themselves. ${ }^{39}$ This conclusion is also supported by a recent study which has demonstrated the absence of direct correlation between microvessel density and the degree of bone marrow involvement by mast cell lesions or the expression of various angiogenic cytokines. ${ }^{39}$

In conclusion, the fibrotic lesions of systemic mastocytosis show abundant mature collagen, reduced vascularization as evidenced by scarce collagen IV, laminin, and CD34 expression, and the paucity of cells expressing low-affinity nerve growth factor receptor and stromal cells showing myofibroblastic differentiation. This pattern of stromal change is uncommonly seen in other bone marrow disorders characterized by fibrosis. These observations suggest that the pathogenetic mechanism leading to fibrosis in systemic mastocytosis may differ from that of other fibrotic neoplasms of the bone marrow. Different levels or various combinations of fibrogenic factors may play an important role and explain the differences. Although most likely involved in the pathogenesis of stromal changes seen in both conditions, the expression of TGF- $\beta$ was found to be much more heterogeneous in cases of systemic mastocytosis than in primary myelofibrosis. ${ }^{40}$ It is also tempting to hypothesize that histamine and tryptase, fibrogenic substances more specifically associated with systemic mastocytosis, may be at least partially responsible for the observed effect. ${ }^{41-43}$ In this context, it has been shown that the D816V-activating KIT mutation seen in systemic mastocytosis is not only capable of causing increased mast cell proliferation and survival, but also can render the mast cells more sensitive to the antigen-IgE immune complex. This in turn enhances mast cell degranulation and increases the levels of histamine and tryptase, which promote collagen I synthesis by the fibroblasts. ${ }^{41}$ That may explain why histamine receptor blockers are a useful adjuvant in the current therapeutic regimens for systemic mastocytosis patients, particularly when used in conjunction with tyrosine kinase inhibitors, which have a partial anti KIT activity, such as dasatinib and PKC412. ${ }^{44,45}$ Finally, the identification of the type of fibrosis in systemic mastocytosis, in conjunction with the cytokines involved, may aid in the selection of an appropriate targeted therapeutic approach, and in monitoring the effects following tyrosine kinase inhibitor treatment.

\section{References}

1 Horny HP, Valent P. Diagnosis of mastocytosis: general histopathological aspects, morphological criteria, and immunohistochemical findings. Leuk Res 2001;25: 543-551.

2 Horny HP, Metcalfe DD, Bennett JM, et al. Mastocytosis. In: Swerdlow SH, Campo E, Harris NL et al. (eds). WHO Classification of Tumours of Haematopoietic and Lymphoid Tissues, 4th edn. IARC Press: Lyon, 2008, pp 58-61.

3 Kanbe N, Kurosawa M, Nagata H, et al. Cord bloodderived human cultured mast cells produce transforming growth factor beta1. Clin Exp Allergy 1999;29: 105-113.

4 Hiragun T, Morita E, Tanaka T, et al. A fibrogenic cytokine, platelet-derived growth factor (PDGF), enhances mast cell growth indirectly via a SCF- and fibroblastdependent pathway. J Invest Dermatol 1998;111:213-217.

5 Hamada H, Vallyathan V, Cool CD, et al. Mast cell basic fibroblast growth factor in silicosis. Am J Respir Crit Care Med 2000;161:2026-2034.

6 Ruoss SJ, Hartmann T, Caughey GH. Mast cell tryptase is a mitogen for cultured fibroblasts. J Clin Invest 1991;88:493-499.

7 Cairns JA, Walls AF. Mast cell tryptase stimulates the synthesis of type I collagen in human lung fibroblasts. J Clin Invest 1997;99:1313-1321.

8 Hatamochi A, Ueki H, Mauch C, et al. Effect of histamine on collagen and collagen m-RNA production in human skin fibroblasts. J Dermatol Sci 1991;2: 407-412.

9 Takeda T, Goto H, Arisawa T, et al. Effect of histamine on human fibroblast in vitro. Arzneimittelforschung 1997;47:1152-1155.

10 Thiele J, Kvasnicka HM, Facchetti F, et al. European consensus on grading bone marrow fibrosis and assessment of cellularity. Haematologica 2005;90: 1128-1132.

11 Watanabe Y. Fine structure of bone marrow stroma. Acta Hematol Jpn 1985;48:1688-1700.

12 Weiss L, Sakai H. The hematopoietic stroma. Am J Anat 1984;170:447-463.

13 Mayani H, Guilbert LJ, Janowska-Wieczorek A. Biology of the hemopoietic microenvironment. Eur J Haematol 1992;49:225-233.

14 Cattoretti G, Schiro R, Orazi A, et al. Bone marrow stroma in humans: anti-nerve growth factor receptor antibodies selectively stain reticular cells in vivo and in vitro. Blood 1993;81:1726-1738.

15 Schmitt-Gräff A, Skalli O, Gabbiani G. Alpha-smooth muscle actin is expressed in a subset of bone marrow stromal cells in normal and pathological conditions. Virchows Arch B Cell Pathol Incl Mol Pathol 1989; 57:291-302.

16 Bonanno E, Ercoli L, Missori P, et al. Homogeneous stromal cell population from normal human adult 
bone marrow expressing alpha-smooth muscle actin filaments. Lab Invest 1994;71:308-315.

17 Klein G, Muller CA, Tillet E, et al. Collagen type VI in the human bone marrow microenvironment: a strong cytoadhesive component. Blood 1995;86:1740-1748.

18 Bentley SA, Alabaster O, Foidart JM. Collagen heterogeneity in normal human bone marrow. Br J Haematol 1981;48:287-291.

19 Reilly JT, Nash JR, Mackie MJ, et al. Immunoenzymatic detection of fibronectin in normal and pathological haematopoietic tissue. $\mathrm{Br} \mathrm{J}$ Haematol 1985;59:497-504.

20 Mayani H. Composition and function of the hemopoietic microenvironment in human myeloid leukemia. Leukemia 1996;10:1041-1047.

21 Lisse I, Hasselbalch H, Junker P. Bone marrow stroma in idiopathic Myelofibrosis and other haematological diseases: an immunohistochemical study. APMIS 1991;99:171-178.

22 Thiele J, Rompcik V, Wagner S, et al. Vascular architecture and collagen type IV in primary myelofibrosis and polycythaemia vera: an immunomorphometric study on trephine biopsies of the bone marrow. Br J Haematol 1992;80:227-234.

23 Fang W, An C, Jiang J, et al. The stromal composition of myelofibrosis: differences between chronic myeloproliferative disorders and metastatic malignancies. Mod Pathol 2003;16:232A.

24 Vega F, Medeiros LJ, Lang WH, et al. The stromal composition of malignant lymphoid aggregates in bone marrow: variations in architecture and phenotype in different B-cell tumours. Br J Haematol 2002;117: 569-576.

25 O'Malley DP, Sen J, Juliar BE, et al. Evaluation of stroma in human immunodeficiency virus/acquired immunodeficiency syndrome-affected bone marrows and correlation with CD4 counts. Arch Pathol Lab Med 2005;129:1137-1140.

26 Caneva L, Soligo D, Cattoretti G, et al. Immunoelectron microscopy characterization of human bone marrow stromal cells with anti-NGFR antibodies. Blood Cells Mol Dis 1995;21:73-85.

27 Lewis MP, Lygoe KA, Nystrom ML, et al. Tumourderived TGF-beta1 modulates myofibroblast differentiation and promotes HGF/SF-dependent invasion of squamous carcinoma cells. Br J Cancer 2004;90: 822-832.

28 Papadopoulos N, Simopoulos C, Kotini A, et al. Differential expression of alpha smooth muscle actin molecule in a subset of bone marrow stromal cells, in b-cell chronic lymphocytic leukemia, autoimmune disorders and normal fetuses. Eur J Gynaecol Oncol 2001;22:447-450.

29 Apaja-Sarkkinen M, Autio-Harmainen H, Alavaikko $\mathrm{M}$, et al. Immunohistochemical study of basement membrane proteins and type III procollagen in myelofibrosis. Br J Haematol 1986;63:571-580.
30 Sasano H, Suzuki T. Pathological evaluation of angiogenesis in human tumor. Biomed Pharmacother 2005;59(Suppl 2):S334-S336.

31 Kvasnicka HM, Thiele J. Bone marrow angiogenesis: methods of quantification and changes evolving in chronic myeloproliferative disorders. Histol Histopathol 2004;19:1245-1260.

32 Arora B, Ho CL, Hoyer JD, et al. Bone marrow angiogenesis and its clinical correlates in myelofibrosis with myeloid metaplasia. Haematologica 2004;89: 1454-1458.

33 Ponzoni M, Savage DG, Ferreri AJ, et al. Chronic idiopathic myelofibrosis: independent prognostic importance of bone marrow microvascular density evaluated by CD105 (endoglin) immunostaining. Mod Pathol 2004;17:1513-1520.

34 Cervantes F, Mesa R, Barosi G. New and old treatment modalities in primary myelofibrosis. Cancer J 2007;13: 377-383.

35 Weinkove R, Reilly JT, McMullin MF, et al. Low-dose thalidomide in myelofibrosis. Haematologica 2008;93:1100-1101.

36 Damaj G, Bernit E, Ghez D, et al. Thalidomide in advanced mastocytosis. Br J Haematol 2008;141: 249-253.

37 Kluin-Nelemans HC, Ferenc V, van Doormaal JJ, et al. Lenalidomide therapy in systemic mastocytosis. Leuk Res 2009;33:e19-e22.

38 Wimazal F, Jordan JH, Sperr WR, et al. Increased angiogenesis in the bone marrow of patients with systemic mastocytosis. Am J Pathol 2002;160:1639-1645.

39 Baek JY, Li CY, Pardanani A, et al. Bone marrow angiogenesis in systemic mast cell disease. J Hematother Stem Cell Res 2002;11:139-146.

40 Baek JY, Tefferi A, Pardanani A, et al. Immunohistochemical studies of c-kit, transforming growth factorbeta, and basic fibroblast growth factor in mast cell disease. Leuk Res 2002;26:83-90.

41 Murota H, Bae S, Hamasaki Y, et al. Emedastine difumarate inhibits histamine induced collagen synthesis in dermal fibroblasts. J Investig Allergol Clin Immunol 2008;18:245-252.

42 Albrecht M, Muller K, Kohn FM, et al. Ionizing radiation induces degranulation of human mast cells and release of tryptase. Int J Radiat Biol 2007;83:535-541.

43 Orfao A, Garcia-Montero AC, Sanchez L, et al. Recent advances in the understanding of mastocytosis: the role of KIT mutations. Br J Haematol 2007;138:12-30.

44 Ustun C, Corless CL, Savage N, et al. Chemotherapy and dasatinib induce long-term hematologic and molecular remission in systemic mastocytosis with acute myeloid leukemia with KIT(D816V). Leuk Res 2009;33:735-741.

45 Aichberger KJ, Sperr WR, Gleixner KV, et al. Treatment responses to cladribine and dasatinib in rapidly progressing aggressive mastocytosis. Eur J Clin Invest 2008;38:869-873. 